\title{
Evaluation of candidate barcoding markers in Orinus (Poaceae)
}

\author{
X. Su${ }^{1,2}$, Y.P. Liu ${ }^{1}$, Z. Chen ${ }^{1}$ and K.L. Chen ${ }^{1}$ \\ ${ }^{1}$ Key Laboratory of Physical Geography and Environmental Process, \\ Qinghai Province, Key Laboratory of Medicinal Animal and Plant Resource, \\ Qinghai-Tibetan Plateau of Qinghai Province, \\ Key Laboratory of Education Ministry of Environments and Resources in The \\ Qinghai-Tibetan Plateau, School of Geography and Life Science, \\ Qinghai Normal University, Xining, Qinghai, China \\ ${ }^{2}$ State Key Laboratory Breeding Base-Key Laboratory of Qinghai Province \\ for Plateau Crop Germplasm Innovation and Utilization, Qinghai University, \\ Xining, Qinghai, China \\ Corresponding author: K.L. Chen \\ E-mail: ck17813@163.com
}

Genet. Mol. Res. 15 (2): gmr.15027714

Received September 23, 2015

Accepted December 7, 2015

Published April 25, 2016

DOI http://dx.doi.org/10.4238/gmr.15027714

\begin{abstract}
Orinus is an alpine endemic genus of Poaceae. Because of the imperfect specimens, high level of intraspecific morphological variability, and homoplasies of morphological characters, it is relatively difficult to delimitate species of Orinus by using morphology alone. To this end, the DNA barcoding has shown great potential in identifying species. The present study is the first attempt to test the feasibility of four proposed DNA barcoding markers (matK, rbcL, trnH-psbA, and ITS) in identifying four currently revised species of Orinus from the Qinghai-Tibetan Plateau (QTP). Among all the single-barcode candidates, the differentiation power was the highest for the nuclear internal transcribed spacer (ITS), while the chloroplast barcodes matK $(\mathrm{M}), \operatorname{rbcL}(\mathrm{R})$, and trnH-psbA $(\mathrm{H})$ could not identify the species. Meanwhile, the differentiation efficiency of the nuclear ITS (I) was
\end{abstract}


also higher than any two- or three-locus combination of chloroplast barcodes, or even a combination of ITS and any chloroplast barcode except $\mathrm{H}+\mathrm{I}$ and $\mathrm{R}+\mathrm{I}$. All the combinations of chloroplast barcodes plus the nuclear ITS, $\mathrm{H}+\mathrm{I}$, and $\mathrm{R}+\mathrm{I}$ differentiated the highest portion of species. The highest differentiation rate for the barcodes or barcode combinations examined here was $100 \%(\mathrm{H}+\mathrm{I}$ and $\mathrm{R}+\mathrm{I})$. In summary, this case study showed that the nuclear ITS region represents a more promising barcode than any maternally inherited chloroplast region or combination of chloroplast regions in differentiating Orinus species from the QTP. Moreover, combining the ITS region with chloroplast regions may improve the barcoding success rate.

Key words: DNA barcoding; Internal transcribed spacer; matK; rbcL; trnH-psbA; Orinus

\section{INTRODUCTION}

DNA barcoding is a molecular tool that uses a short, standardized DNA for fast and accurate species identification (Hebert et al., 2003). This technique has been used in various fields, such as in the discovery of new or cryptic species (Liu et al., 2011), accurate species identification (Feng et al., 2013), and for assessment of biodiversity richness in a given area or community (Valentini et al., 2009).

General requirements for an ideal DNA barcoding are easy amplification in the polymerase chain reaction (PCR), high throughput sequence, a short length to allow bidirectional reading from a single-primer pair, and a considerably higher interspecific genetic variability in comparison to intraspecific variability (Kress et al., 2005). For example, the mitochondrial cytochrome $c$ oxidase subunit $1(\mathrm{CO})$ gene, is highly efficient in differentiating animal species, including amphibians (Vences et al., 2005), birds (Hebert et al., 2004), and fishes (Ward et al., 2009), and is accepted as a standard barcode in animals (Hubert et al., 2008). However, it is not a suitable DNA marker in plants because of low mutation rates (Cho et al., 2004). Chloroplast and nuclear gene fragments with fast rates of evolution have become suitable candidate DNA markers in plants (CBOL Plant Working Group, 2009; Hollingsworth et al., 2009; Li et al., 2011). Based on comprehensive evaluation, the CBOL Plant Working Group (2009) formally recommended the combination of two chloroplast locus, matK + $\mathrm{rbcL}$, as the core barcode for land plants, and trnH-psbA and the nuclear ribosomal ITS as complementary markers to the core barcode (Hollingsworth et al., 2009). Recently, on the basis of a larger collection of samples, Li et al. (2011) proposed that the ITS (or ITS2) should also be included in the core barcode of seed plants.

Orinus Hitchcock, an alpine perennial grass, belonging to Tridentinae of Eragrostideae (Poaceae; Chen and Phillips, 2006), was classified by Hitchcock (1933) based on species $O$. arenicola Hitchcock, which was collected for the first time in the Kashmir region. In 1950s, another species of Orinus, O. anomala Keng ex f. and L. Liou, was discovered by Keng (1959) and validated by Keng and Liou (1960). However, Bor (1960) observed that $O$. arenicola Hitchcock was a synonym of Diplachne thoroldii Stapf ex Hemsley, previously described from Tibet region. This representative species of Orinus was shifted by Bor (1960) to O. thoroldii (Stapf ex Hemsl.) Bor. Subsequently, Tzvelev (1968) classified Cleistogenes 
kokonorica K.S. Hao as Orinus kokonorica (K.S. Hao) Tzvelev. Meanwhile, Zhao and Li (1994) described a new species, O. tibeticus N.X. Zhao, from Tibet. Recently, Zhang and Cai (2008), and Su and Cai (2009) individually reported new species, O. alticulmus L.B. Cai and T.L. Zhang, and O. longiglumis L.B. Cai and X. Su. To date, six species are accepted to exist in the genus Orinus (Zhang and Cai, 2008; Su and Cai, 2009). They are mainly distributed in the Qinghai-Tibetan Plateau and its adjacent regions, 2230-5200 m above sea level. The species delimitation in Orinus is based primarily on morphological characters. Specifically, the paniculate inflorescence shapes (spread vs appressed), spikelet length, floret number of each spikelet, and relative length and covering hairs of lemma and palea are the key features for delimitation at the species level in Orinus. In fact, these characters vary considerably, even in the same population. Moreover, some characters such as covering hairs vary according to different living conditions. In addition, because of the imperfect specimens or their limited numbers, many Orinus species have been described using the existing specimens, resulting in incomplete understanding of morphological variations within the species, which affects the taxa revision by plant taxonomists. Consequently, many taxonomic uncertainties remain with regard to Orinus. Recently, through wide survey of the distribution of each Orinus species, statistical analysis of morphological characters at the population levels, and tentative research using the ITS sequence variation, we established that the six previously recognized species should instead be divided into four species ( $\mathrm{Su}$ et al., 2013).

DNA barcoding can be an alternative tool to taxonomic classification, especially when diagnostic morphological characters are missing (Steven and Subramanyam, 2009). However, differentiating closely related or recently evolved species or taxonomic groups with complicated evolutionary histories may be a challenge for DNA barcoding. In the present study, we used four proposed plant DNA barcoding markers (chloroplast: matK, rbcL, and trnH-psbA; nuclear: ITS) and employed them to delimitate 102 samples, representing the six previously described Orinus species. We aimed to 1) evaluate the technicalities and the differentiation capabilities of these DNA markers at the species levels, alone or in combinations; 2) establish a reference database to facilitate future identification of Orinus species in a given area; 3 ) determine whether the four proposed DNA barcoding regions could be used as molecular supplements for morphology-based taxonomy.

\section{MATERIAL AND METHODS}

\section{Plant samples}

Taxa were chosen based on the Flora of China (Chen and Phillips, 2006) and previous studies (Zhao and Li, 1994; Zhang and Cai, 2008; Su and Cai, 2009). Total 102 samples representing six tentative species (currently revised four species) were sampled in the present study (Table S1 and Figure 1). All the investigated species had been identified and characterized, according to the specimen records in the herbaria and morphologic characters. Multiple (two to six) individuals of each species were collected from different localities. Voucher specimens were deposited in the Herbarium of Molecular Ecology Group, State Key Laboratory of Grassland Agro-Ecosystem, School of Life Science, Lanzhou University, Lanzhou, China (LZU). 


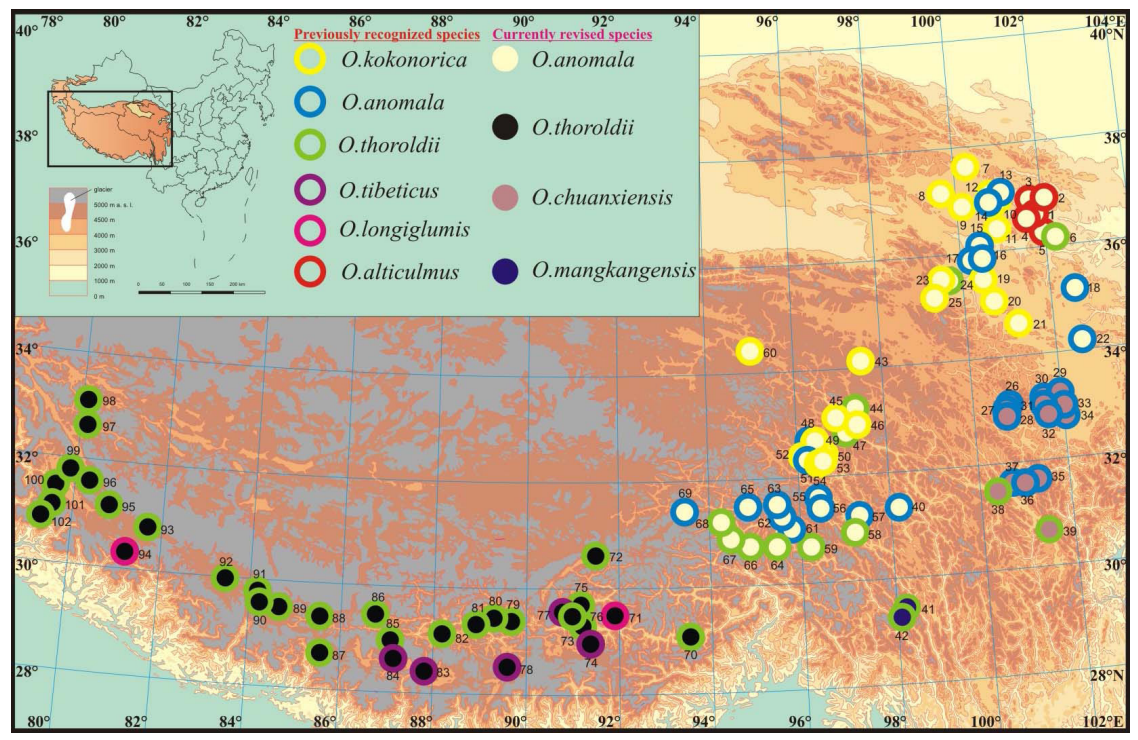

Figure 1. Sampling localities of Orinus surveyed on the Qinghai-Tibetan Plateau.

\section{DNA extraction, amplification, and sequencing}

Total genomic DNA was extracted from each sample using silica-dried leaves, by a modified cetyltrimethylammonium bromide (CTAB) method (Doyle and Doyle, 1987). The forward and reverse primers used for PCR were: 390F and 1326R for matK (Cuénoud et al., 2002); $1 \mathrm{~F}$ and $724 \mathrm{R}$ (or $627 \mathrm{~F}$ and $1504 \mathrm{R}$ as an alternative primer pair), for rbcL (Lledo et al., 1998); trnH and psbA (Sang et al., 1997), for trnH-psbA; and ITS1 and ITS4, respectively, for ITS to amplify both the internal spacers and the 5.8S gene (White et al., 1990). PCR amplifications were performed in $25-\mu \mathrm{L}$ reaction mixtures consisting of $2.5 \mu \mathrm{L} 10 \mathrm{X}$ PCR buffer, $0.25 \mu \mathrm{L}$ TaqDNA polymerase ( $5 \mathrm{U} / \mu \mathrm{L}$; TaKaRa Biotech, Dalian, China), $0.25 \mathrm{mM}$ dNTPs, 0.2 $\mathrm{mM} \mathrm{MgCl}, 2.0 \mu \mathrm{M}$ each primer, and $1.0 \mu \mathrm{L}$ template DNA. The cycling parameters used for matK, trnH-psbA, rbcL, and ITS were $94^{\circ} \mathrm{C}$ for 5 min, followed by 36 cycles of $94^{\circ} \mathrm{C}$ for 50 $\mathrm{s}, 49^{\circ}-58^{\circ} \mathrm{C}$ for $50 \mathrm{~s}$, and $72^{\circ} \mathrm{C}$ for $1 \mathrm{~min}$ or $1 \mathrm{~min}$ and $40 \mathrm{~s}$, with a final extension at $72^{\circ} \mathrm{C}$ for $10 \mathrm{~min}$. The PCR products were purified using a TIAN-quick Midi Purification Kit (Tiangen Biotech, Beijing, China) according to the manufacturer instructions and sequenced in both directions with PCR primers on an ABI 3130xl automated sequencer (Applied Biosystems, Foster City, CA, USA). All the sequences reported in this study were deposited in NCBI and were assigned GenBank accession Nos. KP302391-KP303274 (Table S1).

\section{Data analysis}

Sequence alignments were performed using MUSCLE (Edgar, 2004) and were manually refined using MEGA 5 (Tamura et al., 2011). Insertions/deletions and single nucleotide polymorphisms (SNPs) were identified by DnaSP version 5.0 (Librado and Rozas, 2009). We calculated mean of both intra- and inter-specific Kimura-2 parameter (K2P) distances for each region with pairwise deletion in MEGA 5 (Tamura et al., 2011). To assess 
the efficiency of various barcode combinations on species differentiation, all two- or three-locus combinations as well as the combination of each chloroplast region and ITS were considered; we followed this approach because two or three locus barcodes are often recommended in the published studies (Hollingsworth et al., 2009). To evaluate the success of species differentiation, we applied two different methods: the PWG-Distance and Tree-Building method. The PWG-Distance method (simple pairwise matching for DNA barcoding) recommended by the CBOL Plant Working Group (2009) employed distances calculated from pairwise alignments, counting unambiguous base substitutions only, and pairwise p-distances were calculated using PAUP*4.0b10 (Swofford, 2002); the differentiation was considered successful if the minimum uncorrected interspecific p-distance of a species was larger than its maximum intraspecific distance. When using the tree-building method, a neighbor-joining tree was constructed using the program PAUP*4.0b10 (Swofford, 2002) under the K2P substitution model, and the species were considered successfully differentiated if all the individuals of that species formed a monophyletic group (Li et al., 2011).

\section{RESULTS}

\section{Universality of PCR amplification and sequencing}

Candidate chloroplast barcodes, matK, rbcL, and trnH-psbA were successfully amplified and sequenced with the primers described in previous studies (Table 1). In our study, we observed that both matK and trnH-psbA showed 100\% success in PCR amplification and sequencing, with a single primer pair, whereas only $82 \%$ PCR success was obtained for rbcL because it was much longer. When it was split into two parts, which were then amplified and sequenced with the two primer pairs, the PCR amplification and sequencing success increased to $100 \%$. Meanwhile, amplification and sequencing of the nuclear ITS region with a universal primer combination, resulted in only about $87.2 \%$ amplification and $76.5 \%$ success in sequencing, suggesting low ITS universality. Overall 233 ITS sequences were successfully amplified and sequenced for all the six species, from which 153 sequences had to be excluded from the data analysis because of the presence of one to four base polymorphisms in these sequences. Similarly, 233 sequences were selected and recovered from the chloroplast regions for the six previously recognized species (currently revised to four species).

Table 1. Primer pairs used for amplification and sequencing for DNA barcoding.

\begin{tabular}{|c|c|c|c|}
\hline Region & Primers & Sequences $\left(5^{\prime}-3^{\prime}\right)$ & Reference \\
\hline \multirow[t]{2}{*}{ ITS } & ITS1 & AGAAGTCGTAACAAGGTTTCCGTAGG & \multirow[t]{2}{*}{ White et al., 1990} \\
\hline & ITS4 & TCCTCCGCTTATTGATATGC & \\
\hline \multirow[t]{4}{*}{ rbcL } & rbcL 1F & ATGTCACCACAA ACAGAAACTAAAGC & \multirow[t]{4}{*}{ Lledo et al., 1998} \\
\hline & rbcL 724R & TCGCATGTACCTGCAGTTGC & \\
\hline & rbcL 627F & CATTTATGCGCTGGAGAGACCG & \\
\hline & rbcL 1504R & GAATTACTGATTTCGCAAC & \\
\hline \multirow[t]{2}{*}{ matK } & matK $390 \mathrm{~F}$ & CGATCTATTCATTCAATATTTC & \multirow[t]{2}{*}{ Cuénoud et al., 2002} \\
\hline & matK 1326R & TCTAGCACACGAAAGTCGAAGT & \\
\hline \multirow[t]{2}{*}{$\operatorname{trnH}-\mathrm{psbA}$} & psbAF & GTTATGCATGAACGTAATGCTC & \multirow[t]{2}{*}{ Sang et al., 1997} \\
\hline & $\operatorname{trnH}$ & CGCGCATGGTGGATTCACAAATC & \\
\hline
\end{tabular}




\section{Barcode variation and intra- and inter-specific divergence}

A total of 932 sequences were generated. Variations in the characters of the four candidate DNA barcodes are summarized in Table 2. The aligned lengths of the four candidate DNA markers ranged from $595 \mathrm{bp}$ (trnH-psbA) to $1432 \mathrm{bp}$ (rbcL). Notably, the aligned length of ITS was 632 $\mathrm{bp}$; it contained the maximum number (44) of variable sites including one insertion/deletion $(6.80 \%)$. The aligned length of matK was $843 \mathrm{bp}$ and included 8 variable sites $(0.95 \%)$, which were the lowest. Similarly, the aligned length of rbcL was 1432 bp with 12 variable nucleotides $(0.84 \%)$. It was the longest sequence among the four DNA markers. Furthermore, trnH-psbA matrix was $595 \mathrm{bp}$ long and had 10 variable sites comprising 9 insertions/deletions (1.68\%) and it was one of the shortest sequences. In addition to the four candidate DNA barcodes, ITS demonstrated the highest mean pairwise intra-specific $(0.00078 \pm 0.00040)$ and inter-specific $(0.02297 \pm 0.00520)$ divergences, while trnH-psbA showed the lowest $(0.00012 \pm 0.00012$ and $0.00013 \pm 0.00012$, respectively) divergence, in this study (Table 2 ).

Table 2. Length, recovery rate, variation, and delimitation rate of each DNA region and the combination of the three plastid regions.

\begin{tabular}{l|c|c|c|c|c|c|c|c|c|c|c}
\hline Region & $\begin{array}{c}\text { PCR success } \\
(\%)\end{array}$ & $\begin{array}{c}\text { Sequence } \\
\text { success (\%) }\end{array}$ & $\begin{array}{c}\text { Aligned } \\
\text { length (bp) }\end{array}$ & No. SNPs & $\begin{array}{c}\text { No. } \\
\text { InDels }\end{array}$ & $\begin{array}{c}\text { No. variable } \\
\text { sites (\%) }\end{array}$ & $\begin{array}{c}\text { Intra-specific } \\
\text { divergence }\end{array}$ & $\begin{array}{c}\text { Inter-specific } \\
\text { divergence }\end{array}$ & $\begin{array}{c}\text { Rate (\%) } \\
\text { PWG }\end{array}$ & $\begin{array}{c}\text { Rate (\%) } \\
\text { NJ }\end{array}$ & $\begin{array}{c}\text { No. } \\
\text { sequences }\end{array}$ \\
\hline ITS & 87.2 & 76.5 & 632 & 42 & 1 & $43(6.80)$ & $0.00078 \pm 0.00040$ & $0.02297 \pm 0.00520$ & 100.0 & 100.0 & 220 \\
\hline matK & 100 & 100 & 843 & 8 & 0 & $8(0.95)$ & $0.00052 \pm 0.00031$ & $0.00070 \pm 0.00045$ & 0.0 & 0.0 & 220 \\
\hline rbcL & 100 & 100 & 1432 & 12 & 0 & $12(0.84)$ & $0.00053 \pm 0.00027$ & $0.00118 \pm 0.00055$ & 0.0 & 0.0 & 220 \\
\hline trnH-psbA & 100 & 100 & 595 & 1 & 9 & $10(1.68)$ & $0.00012 \pm 0.00012$ & $0.00013 \pm 0.00012$ & 0.0 & 0.0 & 220 \\
\hline Three cp regions & - & - & 2870 & 21 & 9 & $30(1.05)$ & $0.00045 \pm 0.00020$ & $0.00082 \pm 0.00035$ & 0.0 & 0.0 & 660 \\
\hline
\end{tabular}

$\mathrm{cp}=$ chloroplast; No. SNPs = number of SNPs; No. InDels = number of insertions/deletions; Rate $(\%)=$ percentage successful discrimination of species calculated as the number of species successfully discriminated in relation to the total species; PWG = PWG-distance method; NJ = tree-building method (neighbor-joining tree). Note that statistics for Seq. length, No. SNPs, and No. InDels were derived from an alignment of all (successfully sequenced) specimens.

\section{Species differentiation capability of a single marker or multimarker combinations}

We calculated the differentiation rates for four individual regions and 11 combinations of two, three, or four regions, using the PWG-distance and tree-building methods (Figure 2). The results indicated that any single-chloroplast locus or the combination of all three chloroplast loci could not differentiate the Orinus species, although the combination rbcL + matK was recommended by the CBOL Plant Working Group. However, the nuclear ITS barcode demonstrated the highest species differentiation (tree-building: 100\%; PWG-distance: $100 \%$ ). Therefore, any of the two-marker combinations, in combination with ITS, displayed high species identification. Among these, trnH-psbA + ITS and rbcL + ITS performed the best, with $100 \%$ species differentiation, followed by the combination of matK + ITS with $50 \%$ species differentiation. Adding another plastid marker to these two-marker combinations did not improve the species identification. For example, the combinations of matK $+\mathrm{rbcL}+$ ITS and matK + trnH-psbA + ITS both provided $50 \%$ species differentiation, which was comparable to the two-marker combination matK + ITS. Furthermore, the differentiation efficiency of the combination, rbcL + trnH-psbA + ITS, was 75\% according to the PWG-distance method and $50 \%$ according to the tree-building method. In conclusion, combination of the nuclear ITS region with all three chloroplast regions such as the two-marker combination matK + ITS and the three-marker combination matK $+\mathrm{rbcL}+$ ITS and matK $+\operatorname{trnH}-\mathrm{psbA}+$ ITS provided the same species identification (tree-building: 50\%; PWG-distance: 50\%). 


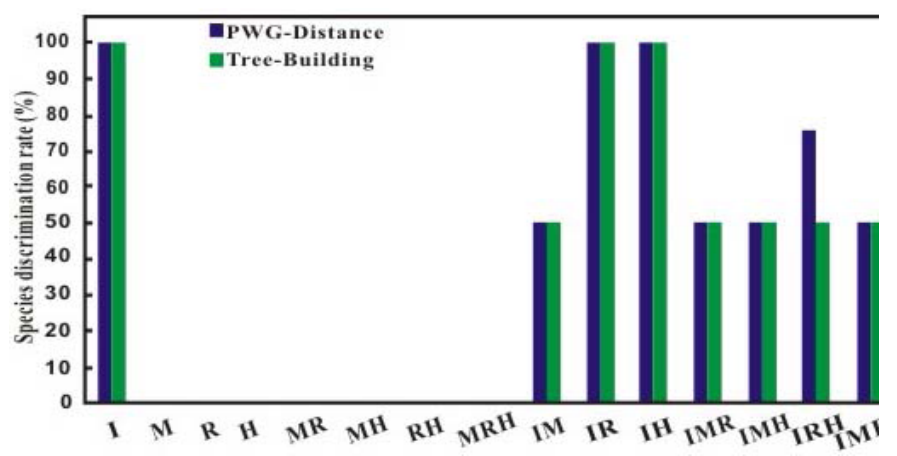

Figure 2. Species discrimination rate of all the tested, single- and multi-locus barcodes, in Orinus. I = ITS; $M=$ matK; $\mathrm{R}=$ rbcL; $\mathrm{H}=$ trnH-psbA.

\section{DISCUSSION}

\section{Universality of the four candidate barcode markers}

Universality of primers is an important criterion for DNA barcoding (Hollingsworth et al., 2009). In the present study, two primer pairs, $1 \mathrm{~F} / 724 \mathrm{R}$ and $627 \mathrm{~F} / 1504 \mathrm{R}$, were tested for rbcL. The latter combination achieved 100\% PCR amplification and sequencing success, suggesting the importance of primer pair selection in the DNA barcoding studies. Similarly, the primer pairs used for matK and trnH-psbA also showed very high levels of primer universality, with $100 \%$ PCR amplification and sequencing success in the genus Orinus. Therefore, the primer pairs 390F/1326R for matK, 627F/1504R for rbcL, and trnH2/psbA for trnH-psbA should be treated as universal primers for Orinus. In comparison, the primer universality of the ITS primer pair ITS1/ ITS4 was low, and only $87.2 \%$ PCR amplification and $76.5 \%$ sequencing success was obtained. Thus, it was unsuitable for DNA barcoding in Orinus species on the basis of the criterion of universality. In previous studies, improvement of the PCR success was attempted through modification of primers such as 5P and 8P (Möller and Cronk, 1997). Obviously, additional ITS primers with high universality were required for DNA barcoding in Orinus species.

\section{Zero efficacy of the three chloroplast locus candidates}

Orinus is an economically and ecologically important genus of grass, but few studies have focused on the delimitation of Orinus since the time it was established. However, the recent development of DNA barcoding provides a novel opportunity in delimitating closely related species. The aim of DNA barcoding is to identify species on the basis of a single-DNA region or a combination of a few DNA regions in the absence of taxonomic knowledge (Hebert et al., 2003). For this reason, the DNA barcode regions should be of short lengths with high recovery rates (success rate for amplifying and sequencing) and have a high species differentiation rate (CBOL Plant Working Group, 2009; Hollingsworth et al., 2009; Li et al., 2011).

Until now, chloroplast regions were regarded as ideal candidates compared to the mitochondrial regions, for plant barcoding by virtue of their faster mutation rate, no recombination, uniparental inheritance, and higher recovery rate (Kress et al., 2005; Li et al., 2011). Testing seven chloroplast loci as barcoding candidates for 907 samples from 550 
species representing the major lineages of land plants, the CBOL Plant Working Group (2009) demonstrated that a single locus, and two-, three-, and seven-locus combinations, respectively, supplied 43-69, 59-75, 65-76, and 73\% resolution at the species level. Based on that, $\mathrm{Li}$ et al. (2011) collected and analyzed 6286 samples representing 1757 species and suggested a similar but slightly lower resolution such as in the case of matK (44.8\%), rbcL (26.4\%), trnH-psbA (45.2\%), matK + rbcL (49.7\%); matK + rbcL + trnH-psbA (62.0\%). Meanwhile, other large scale surveys of chloroplast regions as candidate DNA barcodes, involving fewer samples and species than the above two, have also indicated similar or higher values for differentiate efficacy (Kress et al., 2005).

However, some recent studies demonstrated that single-chloroplast barcoding regions and their different combinations (2-, 3- or 5-loci) had surprisingly lower species differentiation (Hollingsworth et al., 2009; Feng et al., 2013). Similarly, in the present study, we found that all the tested single-chloroplast markers and their multimarker combinations failed completely in differentiating any Orinus species (Figures 2 and 3). This was different from previous studies on other plant groups (CBOL Plant Working Group, 2009; Li et al., 2011; Feng et al., 2013). Among the single-region barcodes, although matK was proposed as a part of the core plant barcode, with a rapid evolutionary rate and a high differentiation rate in previous studies (CBOL Plant Working Group, 2009; Li et al., 2011), none of the four Orinus species sampled in the present study were identified successfully. In addition, the sequence divergence of matK was very low; therefore, they have been suggested to be totally unsuitable DNA barcodes in Orinus. Similarly, both rbcL and trnH-psbA are not good candidate DNA barcodes for Orinus species. With regard to combinations of chloroplast loci, even if the core barcode region combination (matK + rbcL) was recommended by the CBOL Plant Working Group (2009), it could not successfully identify any Orinus species. Based on this, adding trnH-psbA was not able to increase the species differentiation rate. We deduced that the failure to differentiate any species might be attributed to the long life cycle, mixture of sexual and asexual reproduction, and recent rapid speciation of Orinus.

\section{Nuclear ITS region is a very effective DNA barcode in Orinus species differentiation}

ITS usually has more nucleotide variations than any plastid region and has been widely used in phylogeny analysis (Álvarez and Wendel, 2003). Since the nuclear ITS region was suggested as a plant DNA barcode by Kress et al. (2005) because of its rapid evolution and closely related congeneric species differentiation, its use has raised widespread concerns and the region has been tested in numerous studies (Liu et al., 2011). Some recent studies of this region further demonstrated that it had the highest differentiation power at the species level (Li et al., 2011; Feng et al., 2013). In the present study, the successful delimitation rate for the nuclear ITS region was the highest (tree-building: 100\%; PWG-distance: 100\%) among any singlechloroplast region and the combination of all the four candidate DNA markers except for ITS + trnH-psbA (Figure 2). Therefore, these results sufficiently illustrate that the nuclear ITS region should be a more effective DNA barcode than any chloroplast DNA regions in the genus Orinus.

In Orinus, the aligned length of the nuclear ITS region was $632 \mathrm{bp}$ and it contained 43 variable sites $(6.80 \%)$, while that of the three chloroplast DNA regions was 2870 bp and it combined 30 variable sites $(1.05 \%)$; the former being obviously shorter and having a higher mutation rate than the latter (Table 1). Moreover, the nuclear ITS region underwent less introgressions than the three chloroplast DNA regions. 


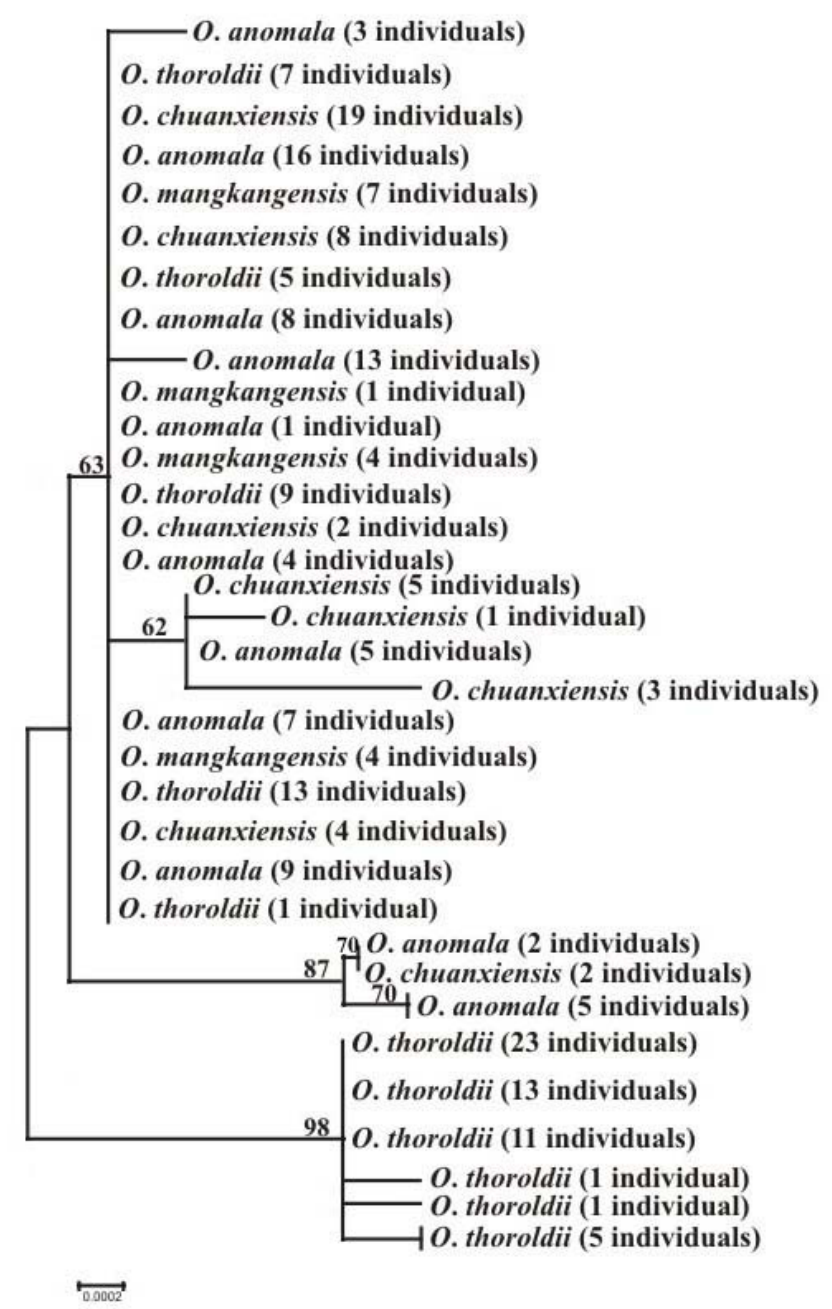

Figure 3. Neighbor-joining tree based on the combination of all three chloroplast regions. Species were successfully delimited using the tree-building method. The combination of all three chloroplast regions cannot differentiate the Orinus species.

Previous studies showed that introgressions could result in transferring of genetic material across species boundaries and genomic components with lower intraspecific gene flow were amenable to introgression. Generally, the nuclear ITS region is biparentally inherited and dispersed by both pollen and seeds, while the chloroplast DNA regions are maternally inherited and dispersed only by seeds (Wang et al., 2011). For genus Orinus, the sexual reproduction should also play an important role in it except for vegetative reproduction. Thus, we thought that the candidate ITS marker should have a higher intraspecific and a lower interspecific gene flow, and higher interspecies differentiation than the three chloroplast markers. Besides, in plants, the nuclear ITS region usually includes multiple-reiterated copies and experiences concerted evolution. During the process, these different copies become almost identical or 
homogenized to the same sequence type as a result of mechanisms such as high frequency unequal crossing over or gene conversion (Álvarez and Wendel, 2003). Therefore, it might have undergone fast lineage sorting and subsequently interspecific differentiation compared with that experienced by speciation genes and linked fragments (Álvarez and Wendel, 2003; Wang et al., 2011). Compared with the three plastid markers, ITS has the advantages of high mutation rate, low introgression, and concerted evolution. Therefore, it has facilitated higher species delimitation efficiency than the chloroplast markers in the genus Orinus (Figures 2 and 4). Naturally, it should be a more effective DNA marker for Orinus, compared to the other plastid markers.

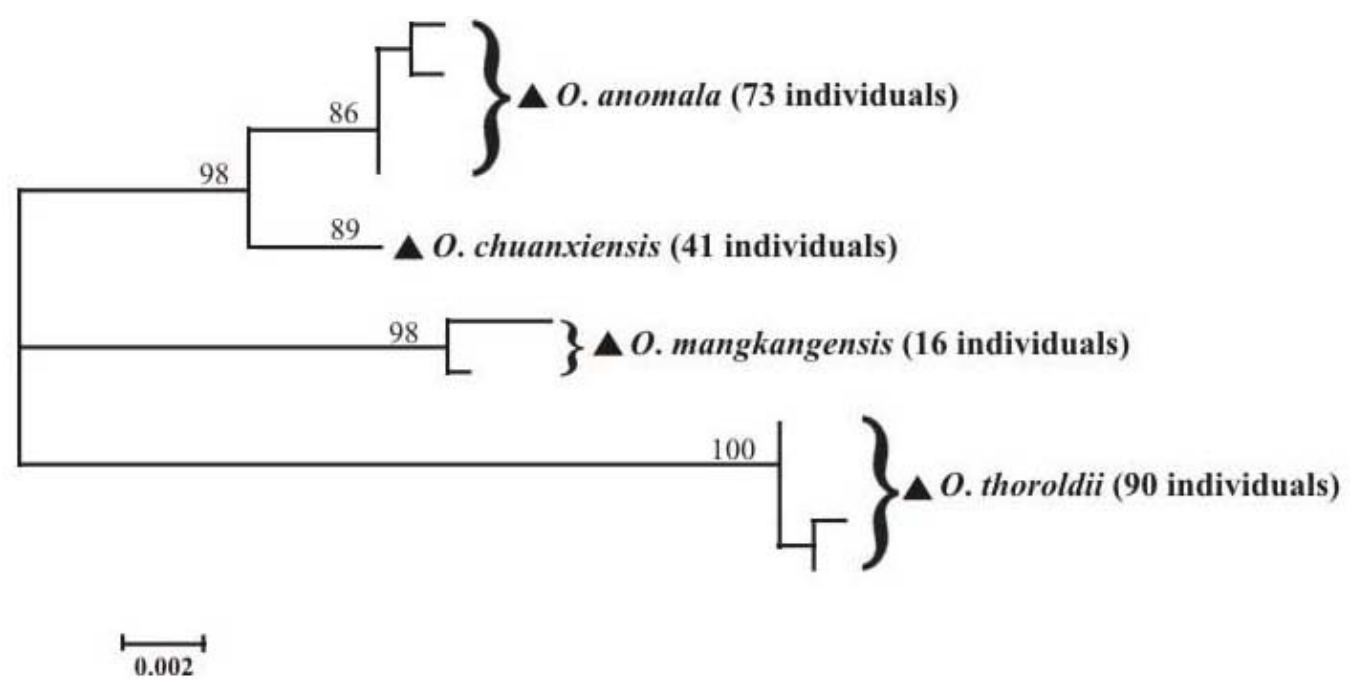

Figure 4. Neighbor-joining tree based on the nuclear transcribed spacer (ITS) regions. Species with solid triangles were successfully delimited using the tree-building method.

\section{Combined nuclear and chloroplast barcode is another effective approach in species delimitation}

Li et al. (2011) previously pointed out that the combinations of the nuclear ITS and chloroplast regions improved species delimitation power and furthered our understanding of the evolutionary processes in plants as a result of their different inheritance modes and track different evolutionary histories. In the present study, we found that the combinations of ITS with any chloroplast marker could improve the species differentiation rate in view of the high species discriminatory power of ITS (Figure 2). This is consistent with the results of previous study of Li et al. (2011) in which it was proposed that ITS should be incorporated into the core barcode for seed plants. This is in agreement with the studies on DNA barcoding of single plant genera such as Alnus (Betulaceae), Holcoglossum (Orichidaceae), and Populus (Salicaceae). Among the seven combinations of the nuclear ITS region and three chloroplast regions, the combination of ITS + trnH-psbA and ITS + rbcL demonstrated the highest species differentiation rate $(100 \%)$, while the combinations of I $+\mathrm{M}, \mathrm{I}+\mathrm{M}+\mathrm{R}, \mathrm{I}+\mathrm{M}+\mathrm{H}, \mathrm{I}+$ $\mathrm{R}+\mathrm{H}$, and $\mathrm{I}+\mathrm{M}+\mathrm{R}+\mathrm{H}$, respectively, successfully delimitated $2 / 2$ (tree-building: $50 \%$; PWG-distance: 50\%; Figures 2 and 5), $2 / 2$ (tree-building: 50\%; PWG-distance: 50\%), 2/3 (tree-building: 50\%; PWG-distance: 75\%), and 2/2 (tree-building: 50\%; PWG-distance: 50\%) 
species out of the four species. These values were higher than those achieved by a single marker or combinations of three candidate chloroplast markers, and were slightly lower than or similar to those derived from the nuclear ITS. Moreover, the combination of ITS + rbcL having the highest $(83.3 \%$ ) discriminatory power (Starr et al., 2009) also showed the highest species differentiation power in Orinus (tree-building: 100\%; PWG-distance: 100\%). Therefore, considered together, we reasoned that the two-marker combinations of ITS + trnH-psbA and ITS + rbcL should be the best choice for barcoding Orinus species. Firstly, this combination provided the highest species differentiation rate among every combination of the nuclear ITS region and the three chloroplast markers (tree-building: 100\%; PWG-distance: 100\%). Secondly, trnH-psbA and rbcL are chloroplast DNA markers, whereas ITS originated from the nuclear genome, and conflicting signals could aid in species differentiation in cases of hybridization ( $\mathrm{Li}$ et al., 2011). Thirdly, trnH-psbA and rbcL are easy to align and show good primer universality.

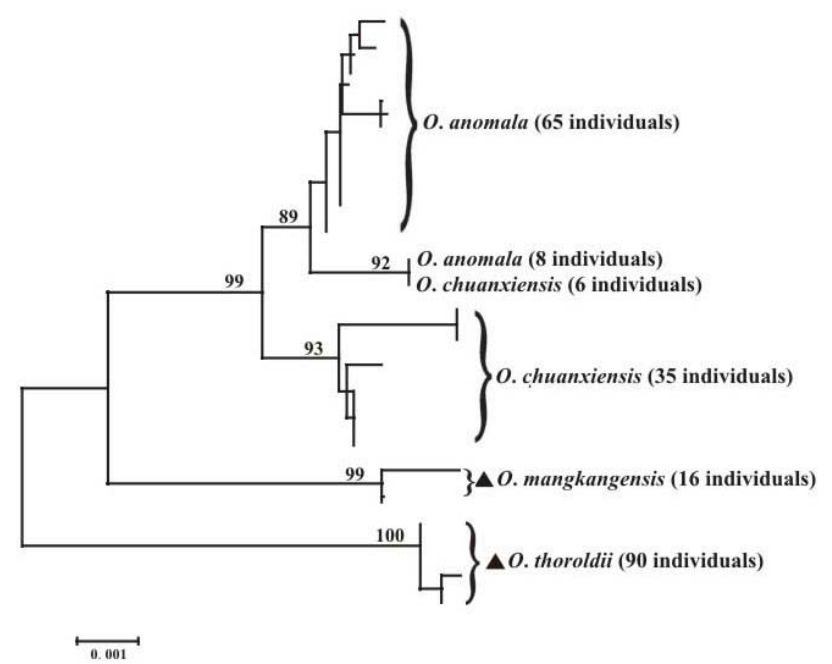

Figure 5. Neighbor-joining tree based on the combination of the nuclear transcribed spacer (ITS) region and one chloroplast (matK) region. Species with solid triangles were successfully delimited using the tree-building method.

\section{Taxonomic implications of DNA barcoding in Orinus species}

Previous studies have suggested that Orinus should consist of six species (Zhao and Li, 1994; Chen and Phillips, 2006; Su and Cai, 2009). This taxonomic treatment has widely been accepted by many scholars. However, were the previously acknowledged species correctly identified and the number of species recognized or delimited for this genus, are some of the many unanswered questions. Therefore, it is imperative to initiate a large scale taxonomic revision of Orinus. Fortunately, because DNA barcoding represents a powerful methodology in testing the existing taxonomic treatments based solely on morphological characters, it may be an appropriate tool for this (Ren et al., 2010). In the present study, we undertook the evaluation of four candidate DNA barcodes of the previously acknowledged species (currently revised to four species), using the nuclear ITS region and three chloroplast regions (matK, rbcL, and trnH-psbA).

It was not unanticipated that the low discriminatory power of the three chloroplast DNA markers demonstrated their limited taxonomic implications in closely related species 
of Orinus (Figures 2 and 3). The plastid non-coding regions (i.e., $\operatorname{trnH}-\mathrm{psbA}, \operatorname{trnT}-\operatorname{trnL}$, and rpl32-trnL) were presumed to have potentially more informative characters than the coding regions including rbcL and matK (Shaw et al., 2007). Meanwhile, the interrelationships among most genera and species remained unresolved, especially in Orinus sampled in this study. Therefore, it was reasonable that the three candidate DNA markers exhibited limited molecular identification capability. This may have been caused by incomplete lineage sorting, interspecific hybridization, and/or introgressions (Hollingsworth et al., 2009). Recently, more highly variable plastid DNA markers were evaluated and suggested for plant barcoding (Dong et al., 2012); we guessed that some of these might provide suitable regions for identifying the Orinus species. With the development of the complete chloroplast genomes of Poaceae, especially Orinus, ideal chloroplast DNA barcoding markers are expected in the future.

The nuclear ITS appeared more promising than the three chloroplast candidate DNA barcodes as a molecular supplement to the morphological taxonomy. The closely related Orinus samples were distinguished into four species by ITS (Figures 2 and 4). This result was in accordance with the morphological identification. It suggested that ITS was significantly able to resolve the Orinus species delimitation in the present study. Therefore, studies based on molecular data (e.g., SSR, AFLP) at the population level are essential to delimit the closely related species boundaries. As discussed by Feng et al. (2013), we also agree with a previous proposal for the improvement of DNA barcoding success rate by the introduction of highly variable loci that are nested under core DNA barcoding markers, such as by building a tiered DNA barcoding system. Such a barcoding system would be especially valuable for economically and ecologically important genera, i.e., Orinus, Populus, and Picea. Based on our results, we suggest that the nuclear ITS or the combination of ITS + trnH-psbA and ITS + rbcL should be the core barcodes for Orinus. In conclusion, we think that the previously reported taxonomy of Orinus species urgently needs further revisions and the currently revised taxonomy demands further verification with the help of a tiered DNA barcoding system.

\section{Conflicts of interest}

The authors declare no conflict of interest.

\section{ACKNOWLEDGMENTS}

Research supported by the National Natural Science Foundation of China (\#31260052), the National Natural Science Foundation of Qinghai Province (\#2014-ZJ-947Q), the Key Laboratory of Medicinal Animal and Plant Resource of Qinghai-Tibetan Plateau of Qinghai Province (\#2015-Z-Y0b), and the State Key Laboratory Breeding Base-Key Laboratory of Qinghai Province for Plateau Crop Germplasm Innovation and Utilization (\#2013-01).

\section{REFERENCES}

Álvarez I and Wendel JF (2003). Ribosomal ITS sequences and plant phylogenetic inference. Mol. Phylogenet. Evol. 29: 417-434. http://dx.doi.org/10.1016/S1055-7903(03)00208-2

Bor NL (1960). The Grasses of Burma, Ceylon, India and Pakistan. Pergamon Press, Oxford and New York.

CBOL Plant Working Group (2009). A DNA barcode for land plants. Proc. Natl. Acad. Sci. USA 106: 12794-12797. http:// dx.doi.org/10.1073/pnas.0905845106

Chen SL and Phillips SM (2006). Orinus (Poaceae). In: Wu ZY, Raven PH and Hong DY, eds. Flora of China. Science Press, Beijing, and Missouri Botanical Garden Press, St. Louis 22: 464-465. 
Cho Y, Mower JP, Qiu YL and Palmer JD (2004). Mitochondrial substitution rates are extraordinarily elevated and variable in a genus of flowering plants. Proc. Natl. Acad. Sci. USA 101: 17741-17746. http://dx.doi.org/10.1073/ pnas.0408302101

Cuénoud P, Savolainen V, Chatrou LW, Powell M, et al. (2002). Molecular phylogenetics of Caryophyllales based on nuclear 18S rDNA and plastid rbcL, atpB, and matK DNA sequences. Am. J. Bot. 89: 132-144. http://dx.doi. org/10.3732/ajb.89.1.132

Dong W, Liu J, Yu J, Wang L, et al. (2012). Highly variable chloroplast markers for evaluating plant phylogeny at low taxonomic levels and for DNA barcoding. PLoS One 7: e35071. http://dx.doi.org/10.1371/journal.pone.0035071

Doyle JJ and Doyle JL (1987). A rapid DNA isolation procedure for small quantities of fresh leaf tissue. Phytochem. Bull. 19: $11-15$.

Edgar RC (2004). MUSCLE: multiple sequence alignment with high accuracy and high throughput. Nucleic Acids Res. 32: 1792-1797. http://dx.doi.org/10.1093/nar/gkh340

Feng J, Jiang D, Shang H, Dong M, et al. (2013). Barcoding poplars (Populus L.) from western China. PLoS One 8: e71710. http://dx.doi.org/10.1371/journal.pone.0071710

Hebert PD, Cywinska A, Ball SL and deWaard JR (2003). Biological identifications through DNA barcodes. Proc. Biol. Sci. 270: 313-321. http://dx.doi.org/10.1098/rspb.2002.2218

Hebert PDN, Stoeckle MY, Zemlak TS and Francis CM (2004). Identification of birds through DNA barcodes. PLoS Biol. 2: e312. http://dx.doi.org/10.1371/journal.pbio.0020312

Hitchcock AS (1933). New grasses from Kashmir. J. Wash. Acad. Sci. 23: 134-136.

Hollingsworth ML, Andra Clark A, Forrest LL, Richardson J, et al. (2009). Selecting barcoding loci for plants: evaluation of seven candidate loci with species-level sampling in three divergent groups of land plants. Mol. Ecol. Resour. 9: 439-457. http://dx.doi.org/10.1111/j.1755-0998.2008.02439.x

Hubert N, Hanner R, Holm E, Mandrak NE, et al. (2008). Identifying Canadian freshwater fishes through DNA barcodes. PLoS One 3: e2490. http://dx.doi.org/10.1371/journal.pone.0002490

Keng PC and Liou L (1960). A study on the tribe Eragrostideae and its two genera new to China. Acta Bot. Sin. 9: 48-75.

Keng YL (1959). Orinus Hitchcock. In: Flora Illustralis Plantarum Primarum Sinicarum Gramineae (Keng YL, ed). Science Press, Beijing, 283-286.

Kress WJ, Wurdack KJ, Zimmer EA, Weigt LA, et al. (2005). Use of DNA barcodes to identify flowering plants. Proc. Natl. Acad. Sci. USA 102: 8369-8374. http://dx.doi.org/10.1073/pnas.0503123102

Li DZ, Gao LM, Li HT, Wang H, et al.; China Plant BOL Group (2011). Comparative analysis of a large dataset indicates that internal transcribed spacer (ITS) should be incorporated into the core barcode for seed plants. Proc. Natl. Acad. Sci. USA 108: 19641-19646. http://dx.doi.org/10.1073/pnas.1104551108

Librado P and Rozas J (2009). DnaSP v5: a software for comprehensive analysis of DNA polymorphism data. Bioinformatics 25: 1451-1452. http://dx.doi.org/10.1093/bioinformatics/btp187

Liu J, Möller M, Gao LM, Zhang DQ, et al. (2011). DNA barcoding for the discrimination of Eurasian yews (Taxus L., Taxaceae) and the discovery of cryptic species. Mol. Ecol. Resour. 11: 89-100. http://dx.doi.org/10.1111/j.17550998.2010.02907.x

Lledo MD, Crespo MB, Cameron KM, Fay MF, et al. (1998). Systematics of Plumbaginaceae based upon cladistic analysis of rbcL sequence data. Syst. Bot. 23: 21-29. http://dx.doi.org/10.2307/2419571

Möller M and Cronk Q (1997). Origin and relationships of Saintpaulia (Gesneriaceae) based on ribosomal DNA internal transcribed spacer (ITS) sequences. Am. J. Bot. 84: 956-965. http://dx.doi.org/10.2307/2446286

Ren BQ, Xiang XG and Chen ZD (2010). Species identification of Alnus (Betulaceae) using nrDNA and cpDNA genetic markers. Mol. Ecol. Resour. 10: 594-605. http://dx.doi.org/10.1111/j.1755-0998.2009.02815.x

Sang T, Crawford D and Stuessy T (1997). Chloroplast DNA phylogeny, reticulate evolution, and biogeography of Paeonia (Paeoniaceae). Am. J. Bot. 84: 1120-1136. http://dx.doi.org/10.2307/2446155

Shaw J, Lickey EB, Schilling EE and Small RL (2007). Comparison of whole chloroplast genome sequences to choose noncoding regions for phylogenetic studies in angiosperms: the tortoise and the hare III. Am. J. Bot. 94: 275-288. http://dx.doi.org/10.3732/ajb.94.3.275

Starr JR, Naczi RF and Chouinard BN (2009). Plant DNA barcodes and species resolution in sedges (Carex, Cyperaceae). Mol. Ecol. Resour. 9 (Suppl s1): 151-163. http://dx.doi.org/10.1111/j.1755-0998.2009.02640.x

Steven GN and Subramanyam R (2009). Testing plant barcoding in a sister species complex of pantropical Acacia (Mimosoideae, Fabaceae). Mol. Ecol. Resour. 9 (Suppl s1): 172-180. http://dx.doi.org/10.1111/j.17550998.2009.02642.x

Su X and Cai LB (2009). Orinus longiglumis (Poaceae: Chloridoideae), a new species from Xizang (Tibet), China. Ann. Bot. Fenn. 46: 143-147. http://dx.doi.org/10.5735/085.046.0211 
Su X, Yue W and Liu JQ (2013). Germplasm collection and preservation of Orinus (Poaceae) in the Qinghai-Tibet Plateau. Plant Diversity Resour. 35: 343-347.

Swofford DL (2002). PAUP*: phylogenetic analysis using parsimony (*and other methods), version 4. Sinauer Associates, Sunderland, MA.

Tamura K, Peterson D, Peterson N, Stecher G, et al. (2011). MEGA5: molecular evolutionary genetics analysis using maximum likelihood, evolutionary distance, and maximum parsimony methods. Mol. Biol. Evol. 28: 2731-2739. http://dx.doi.org/10.1093/molbev/msr121

Tzvelev NN (1968). Plantae Asiae Centralis (Vol. 4). Nauka Publishers, Leningrad, 112.

Valentini A, Pompanon F and Taberlet P (2009). DNA barcoding for ecologists. Trends Ecol. Evol. (Amst.) 24: 110-117. http://dx.doi.org/10.1016/j.tree.2008.09.011

Vences M, Thomas M, Bonett RM and Vieites DR (2005). Deciphering amphibian diversity through DNA barcoding: chances and challenges. Philos. Trans. R. Soc. Lond. B Biol. Sci. 360: 1859-1868. http://dx.doi.org/10.1098/ rstb.2005.1717

Wang Q, Yu QS and Liu JQ (2011). Are nuclear loci ideal for barcoding plants? A case study of genetic delimitation of two sister species using multiple loci and multiple intraspecific individuals. J. Syst. Evol. 49: 182-188. http://dx.doi. org $/ 10.1111 / \mathrm{j} .1759-6831.2011 .00135 . \mathrm{x}$

Ward RD, Hanner R and Hebert PD (2009). The campaign to DNA barcode all fishes, FISH-BOL. J. Fish Biol. 74: 329 356. http://dx.doi.org/10.1111/j.1095-8649.2008.02080.x

White TJ, Bruns T, Lee S and Taylor J (1990). Amplification and direct sequencing of fungal ribosomal RNA genes for phylogenetics. In: PCR protocols: A guide to methods and applications (Innis M, Gelfand D, Sninsky J, White TJ, eds.). Academic Press, San Diego, CA, 315-322.

Zhang TL and Cai LB (2008). A New Species of Orinus (Poaceae) from Qinghai, China. Novon 18: 275-278. http://dx.doi. org $/ 10.3417 / 2006117$

Zhao NX and Li MF (1994). New taxa and new recording species of Gramineae from Tibet. Acta Bot. Yunnanica 16: 228-230.

\section{Supplementary material}

Table S1. Samples for testing potential barcode. 